\title{
HIV and antiretroviral therapy in the brain: neuronal injury and
}

\section{repair}

\author{
Ronald Ellis*, Dianne Langford, Eliezer Masliah, Igor Grant, Robert Heaton, \\ Joseph Wong, Davey Smith, Scott Letendre and Allen McCutchan
}

Address: Department of neurosciences, University of California, San Diego, CA, USA

Email: Ronald Ellis* - roellis@ucsd.edu

* Corresponding author

from Infectious diseases of the nervous system: pathogenesis and worldwide impact Paris, France. 10-13 September 2008

Published: 23 September 2008

BMC Proceedings 2008, 2(Suppl I):SI3

This abstract is available from: http://www.biomedcentral.com/I753-656I/2/SI/SI3

(c) 2008 Ellis et al; licensee BioMed Central Ltd.

Worldwide over 30 million people are infected with human immunodeficiency virus (HIV-1). Although HIV is both neurotropic and neurovirulent, the spectrum of HIVassociated neurocognitive disorders (HAND) remains under-recognized. Combination antiretroviral therapy has improved the health of millions of those living with HIV, yet antiretroviral drugs show highly variable and often inadequate penetration into the CNS. As a result, patients' quality of life continues to be diminished by milder, residual neurocognitive impairments that have not been effectively addressed. HIV causes synaptodendritic neuronal injury that can be measured post-mortem and is a likely source of neurocognitive decline. By carefully selecting specific antiretrovirals and supplementing them with neuroprotective agents, physicians might be able to facilitate innate CNS repair, promoting enhanced synaptodendritic plasticity, neural function and clinical neurological status. 